\title{
Source Enumeration via Toeplitz Matrix Completion
}

\author{
Vaibhav Garg ${ }^{1}$, Pere Giménez-Febrer ${ }^{1,2}$, Alba Pagès-Zamora ${ }^{2}$ and Ignacio Santamaria ${ }^{1}$ \\ ${ }^{1}$ Dept. of Communications Engineering, Universidad de Cantabria, Spain \\ 2 SPCOM Group, Universitat Politècnica de Catalunya-Barcelona Tech, Spain \\ Email: \{vaibhav.garg, gimenezpj\}@unican.es, alba.pages@upc.edu, i.santamaria@unican.es
}

\begin{abstract}
This paper addresses the problem of source enumeration by an array of sensors in the presence of noise whose spatial covariance structure is a diagonal matrix with possibly different variances, referred to non-iid noise hereafter, when the sources are uncorrelated. The diagonal terms of the sample covariance matrix are removed and, after applying Toeplitz rectification as a denoising step, the signal covariance matrix is reconstructed by using a low-rank matrix completion method adapted to enforce the Toeplitz structure of the sought solution. The proposed source enumeration criterion is based on the Frobenius norm of the reconstructed signal covariance matrix obtained for increasing rank values. As illustrated by simulation examples, the proposed method performs robustly for both small and large-scale arrays with few snapshots, i.e. small-sample regime.
\end{abstract}

Index Terms-Array processing, model order estimation, source enumeration, matrix completion, Toeplitz rectification.

\section{INTRODUCTION}

Source enumeration is a classical problem in array signal processing which consists in estimating the number of signals received by an array of sensors. It has applications in numerous fields such as wireless communications, radar, biomedical and geophysical signal processing [1], [2]. Classical approaches to solve this problem are based on information-theoretic criteria [3]-[6], which use order fitting rules based on functions of the eigenvalues of the sample covariance matrix (SCM) penalized by the model complexity. These criteria are derived under a white noise model assumption and their performance degrades when the number of snapshots is relatively small in comparison to the number of antennas, the so-called smallsample regime. Different methods have been proposed for order estimation in the small-sample regime, such as those based on random matrix theory [7]-[9], an exponential fitting test of eigenvalues [10], and bootstrapping [11]. These methods, however, are designed for white noise, and usually provide poor results under non-iid noises. Recently, a subspace averaging (SA) order estimation method has been proposed for white noise [12], [13], and then extended to account for noniid noise models in [14]. The SA method provides good results with low sample support, but it requires high-dimensional scenarios, i.e. large arrays to work satisfactorily since otherwise the averaging procedure is not effective. When the noise covariance matrix is diagonal with unknown elements (a model

This work was supported by the Ministerio de Economía y Competitividad (MINECO) of Spain, and AEI/FEDER funds of the E.U., under grants TEC2016-75067-C4-4-R (CARMEN), PID2019-104958RB-C43/C41 (ADELE) and BES-2017-080542. that becomes relevant in situations with uncalibrated receivers or due to hardware nonidealities), estimating the number of sources is equivalent to estimating the number of common factors in a multivariate factor analysis problem, for which several methods have been proposed in the statistics literature [15], [16]. Algorithms to maximize the likelihood function for this problem can be found in [17], [18]. But again, these methods perform poorly in the small-sample regime.

In this paper we propose a method for enumerating sources in the presence of non-iid noises that performs satisfactorily in a wide number of scenarios, including the small-sample regime both with large and short arrays, and low signal-to-noise-ratio (SNR) environments. The method obtains an estimate of the signal covariance matrix that is i) Toeplitz, and ii) low-rank. To this end, we use a low-rank matrix completion (MC) method that includes an additional regularization term to enforce a Toeplitz structure in the solution. The MC algorithm takes as input a denoised version of the SCM where the elements along the main diagonal have been removed. An additional denoising process known as Toeplitz rectification [19], [20] is used to find a better estimate of the signal covariance matrix. Finally, we propose an order estimation criterion which is based on the Frobenius norm of the matrices reconstructed for increasing rank values.

Low-rank matrix completion methods have been used before in array signal processing problems. In [21], for instance, $\mathrm{MC}$ is used for direction of arrival (DOA) estimation, when the number of sources exceeds the number of sensors. An iterative reweighted nuclear norm minimization method is used in [22] for DOA estimation with nested arrays. The case of non-iid noises is considered in [23], where matrix completion algorithms are used to reconstruct the zeroed entries of the SCM along its diagonal. All these methods, however, address the DOA estimation problem and assume that the number of sources is known. Differently from these works, in this paper we exploit the eigenvalue sparsity provided by MC algorithms to devise a novel order estimation criterion.

Notation. The superscripts $(\cdot)^{T}$ and $(\cdot)^{H}$ denote transpose and Hermitian, respectively. The trace and Frobenius norm of a matrix $\mathbf{B}$ will be denoted, respectively, as $\operatorname{tr}(\mathbf{B})$ and $\|\mathbf{B}\|_{F}$, whereas $|\cdot|$ denotes the norm of a complex number. Furthermore, $\mathbf{x} \sim \mathcal{C N}_{n}(\mathbf{0}, \mathbf{R})$ denotes a complex Gaussian vector in $\mathbb{C}^{n}$ with zero mean and covariance $\mathbf{R}$. 


\section{Problem Statement}

Let us consider $K$ narrowband signals impinging on a uniform linear array (ULA) with $M$ antennas. The received signal is

$$
\mathbf{x}[n]=\left[\mathbf{a}\left(\theta_{1}\right), \ldots, \mathbf{a}\left(\theta_{K}\right)\right] \mathbf{s}[n]+\mathbf{e}[n]=\mathbf{A} \mathbf{s}[n]+\mathbf{e}[n],
$$

where $\mathbf{a}\left(\theta_{k}\right)=\left[\begin{array}{llll}1, & e^{-j \theta_{k}}, & \ldots & e^{-j \theta_{k}(M-1)}\end{array}\right]^{T}$ is the $M \times 1$ complex array response for the $k$ th source, with unknown DOA $\theta_{k}$. The signals are assumed to be uncorrelated and are modelled as $\mathbf{s}[n] \sim \mathcal{C N}_{K}(\mathbf{0}, \Psi)$, where $\boldsymbol{\Psi}$ is a diagonal matrix. The noises $\mathbf{e}[n] \sim \mathcal{C N}_{M}(\mathbf{0}, \boldsymbol{\Sigma})$, are assumed to be temporarily white, uncorrelated across antennas with different variances at each sensor (non-iid). Therefore, the noise covariance matrix is $\boldsymbol{\Sigma}=\operatorname{diag}\left(\sigma_{1}^{2}, \sigma_{2}^{2}, \ldots, \sigma_{M}^{2}\right)$, where $\sigma_{m}^{2}$ is the noise variance at the $m$-th sensor. The noise variances are modeled as uniformly distributed independent random variables: $\sigma_{m}^{2} \sim \mathcal{U}\left[\sigma^{2}(1-\epsilon), \sigma^{2}(1+\epsilon)\right]$, where $\sigma^{2}$ is a common noise variance and $0 \leq \epsilon \leq 1$ allows us to control the spatial non-whiteness of the noise. Notice that for $\epsilon=0$ the noise is spatially white with covariance matrix $\boldsymbol{\Sigma}=\sigma^{2} \mathbf{I}$.

From the signal model (1), the covariance matrix is

$$
\mathbf{R}=\mathbb{E}\left[\mathbf{x}[n] \mathbf{x}^{H}[n]\right]=\mathbf{R}_{s}+\mathbf{\Sigma},
$$

where $\mathbf{R}_{s}=\mathbf{A} \boldsymbol{\Psi} \mathbf{A}^{H}$ is the signal covariance matrix. If $N$ snapshots are collected in the data matrix matrix $\mathbf{X}=$ $\left[\begin{array}{lll}\mathbf{x}[1] & \ldots & \mathbf{x}[N]\end{array}\right]$, then the sample covariance matrix is

$$
\hat{\mathbf{R}}=\frac{1}{N} \sum_{n=1}^{N} \mathbf{x}[n] \mathbf{x}^{H}[n]=\frac{1}{N} \mathbf{X X}^{H} .
$$

The source enumeration problem consists in estimating $K$ from $\mathbf{X}$ or $\hat{\mathbf{R}}$.

\section{ORder Estimation Via TOEPLitz MATRIX COMPLETION}

The proposed order determination criterion builds upon the reconstruction of the signal covariance matrix $\mathbf{R}_{s}$ in (2) for increasing values of its rank. To this end, we apply a matrix completion (MC) approach, which is described in the following subsection. Then, we introduce the proposed order fitting rule, which is based on the Frobenius norm of the reconstructed matrices.

\section{A. Toeplitz Matrix Completion}

The signal covariance matrix $\mathbf{R}_{s}$ is Toeplitz and low-rank. These two properties are not fulfilled by the sample covariance $\hat{\mathbf{R}}$, which is symmetric (but non-Toeplitz) and full-rank. In addition, since $\boldsymbol{\Sigma}$ in (2) is diagonal, the off-diagonal terms of $\mathbf{R}$ are unaffected by the noise covariance matrix. Therefore, the diagonal entries of $\hat{\mathbf{R}}$ will be more affected by the noise. Matrix completion algorithms can then be used to reconstruct the low-rank signal covariance matrix from the off-diagonal terms of $\hat{\mathbf{R}}$.
Let $\Omega=\{(i, j): i \neq j, i=1, \ldots, M, j=1, \ldots, M\}$ be the set of indices for the off-diagonal entries of $\hat{\mathbf{R}}$. According to [24], we can recover $\mathbf{R}_{s}$ by solving

$$
\begin{array}{ll}
\min _{\mathbf{R}_{s} \in \mathbb{C}^{M \times M}} & \left\|\mathbf{R}_{s}\right\|_{*} \\
\text { subject to } & \left\|P_{\Omega}\left(\mathbf{R}_{s}-\hat{\mathbf{R}}\right)\right\|_{F} \leq \eta
\end{array}
$$

where $P_{\Omega}$ denotes the projection operator that sets to zero the entries with indices not belonging to $\Omega$ and leaves the rest unchanged, and $\left\|\mathbf{R}_{s}\right\|_{*}$ denotes the nuclear norm of $\mathbf{R}_{s}$.

Due to the fact that we are dealing with a limited number of snapshots, the nonzero entries in $P_{\Omega}(\hat{\mathbf{R}})$ are still noisy [19] and thus recovering the full $\mathbf{R}_{s}$ via (4) might yield unreliable estimates in the low-sample regime. Therefore, we propose to use a denoising step called Toeplitz rectification [19], [20], [25] before applying matrix completion, and enforce the Toeplitz structure in the reconstruction process.

An unbiased estimator of sample covariance with Toeplitz structure is obtained by averaging its entries along each subdiagonal as [25]

$$
\hat{\mathbf{R}}_{\text {toep }}(i, j)=\frac{1}{M-|i-j|} \sum_{l-m=i-j} \hat{\mathbf{R}}(l, m) .
$$

This process reduces the noise in the off-diagonal terms and provides a better reconstruction of the signal covariance matrix.

After Toeplitz correction, we proceed to perform matrix completion by means of matrix factorization [26]. Since the signal covariance matrix is symmetric, we can factorize it as $\mathbf{R}_{s}=\mathbf{W} \mathbf{W}^{H}$, where $\mathbf{W} \in \mathbb{C}^{M \times p}$ and $p$ is a fixed value that limits the rank of the reconstructed matrix. Then, using

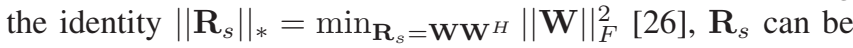
estimated by solving the following optimization problem

$$
\begin{gathered}
\hat{\mathbf{W}}=\underset{\mathbf{W} \in \mathbb{C}^{M \times p}}{\operatorname{argmin}}\left\|P_{\Omega}\left(\hat{\mathbf{R}}_{\text {toep }}-\mathbf{W} \mathbf{W}^{H}\right)\right\|_{F}^{2}+\mu\|\mathbf{W}\|_{F}^{2} \\
\text { s.t. } \quad \mathbf{W} \mathbf{W}^{H} \in \mathcal{T}
\end{gathered}
$$

where $\mu$ is a regularization parameter and the constraint restricts the solution to a set $\mathcal{T}$, which we define as the set of Toeplitz matrices in $\mathbb{C}^{M \times M}$. Note that the term weighted by $\mu$ regulates the nuclear norm of the solution, hence leading to a sparse eigenvalue distribution as $\mu$ grows. An approximate solution to (6) can be obtained by introducing the Toeplitz constraint in the form of an additional regularization term as follows

$$
\begin{aligned}
\hat{\mathbf{W}}=\underset{\mathbf{W} \in \mathbb{C}^{M \times p}}{\operatorname{argmin}} \sum_{(i, j) \in \Omega}\left|\hat{\mathbf{R}}_{\text {toep }}(i, j)-\mathbf{w}_{i}^{H} \mathbf{w}_{j}\right|^{2}+\mu \sum_{i=1}^{M}\left\|\mathbf{w}_{i}\right\|_{2}^{2} \\
+\alpha \sum_{m=0}^{M-1} \sum_{i=1}^{M-m-1}\left|\mathbf{w}_{i}^{H} \mathbf{w}_{i+m}-\mathbf{w}_{i+1}^{H} \mathbf{w}_{i+1+m}\right|^{2}
\end{aligned}
$$

where $\mathbf{w}_{i}^{H}$ is the $i$-th row vector of $\mathbf{W}$, and $\alpha$ is a regularization scalar. The solution $\hat{\mathbf{R}}_{m c}=\hat{\mathbf{W}} \hat{\mathbf{W}}^{H}$ can be obtained by iteratively optimizing over each $\mathbf{w}_{i}$ until convergence, at a cost of $\mathcal{O}\left(p^{3}\right)$ per iteration. 


\section{B. Order Estimation Criterion}

The main insight for the proposed order estimation criterion is that, due to the eigenvalue sparsity enforced by the MC algorithm, as long as $p$ (the rank used in the factorization) is larger than $K$, the reconstructed signal covariance matrix $\hat{\mathbf{R}}_{m c}$ should not change significantly. This intuition is corroborated in Fig. 1a which shows how the Frobenius norm of $\hat{\mathbf{R}}_{m c}$ changes with $p$. It can be observed that the norm grows until $p=K$, but once $p$ exceeds $K$ it is almost constant. This suggests to use the difference function $D(p)$ defined as

$$
D(p)=\left\|\hat{\mathbf{R}}_{m c}(p)\right\|_{F}^{2}-\left\|\hat{\mathbf{R}}_{m c}(p-1)\right\|_{F}^{2}
$$

where $\hat{\mathbf{R}}_{m c}(p)$ denotes the reconstructed signal covariance matrix at a particular value of $p$. Since $D(p)$ will take very small values for $p>K$, finding the position at which this sharp decline change occurs will yield an order estimate. To this end, we propose the following criterion

$$
\hat{k}_{m c}=\underset{1 \leq p \leq p_{\max }}{\operatorname{argmax}} \frac{D(p)}{D(p+1)+\delta}
$$

where $p_{\max }$ is an overestimation of $K$ and $\delta$ is a small constant to avoid numerical issues. Fig. 1b shows $\frac{D(p)}{D(p+1)+\delta}$ for different $K$, where each line has been normalized by its maximum value to enhance visibility. We observe that the peaks are positioned at $p=K$, thus evidencing that the chosen criterion is adequate. A summary of the order estimation via Toeplitz matrix completion (TMC) algorithm is shown in Algorithm 1.
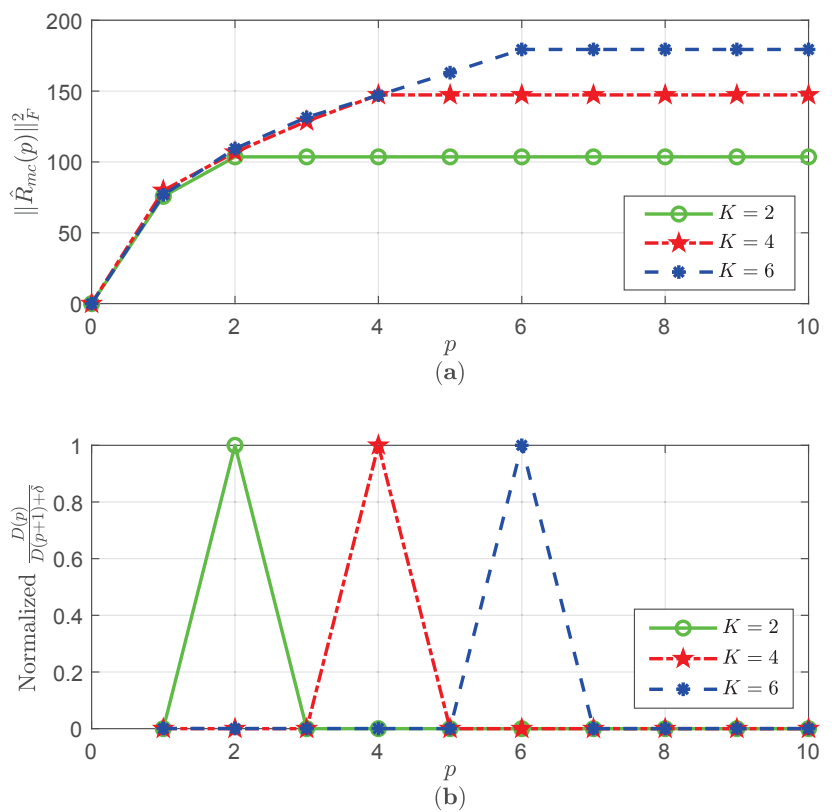

Fig. 1: (a) $\left\|\hat{\mathbf{R}}_{m c}(p)\right\|_{F}^{2}$ vs. $p$, and (b) Normalized $\frac{D(p)}{D(p+1)+\delta}$ vs. $p$ for $M=100, N=150, \epsilon=0.5, \mathrm{SNR}=-10 \mathrm{~dB}$ and sources are separated by $10^{\circ}$.

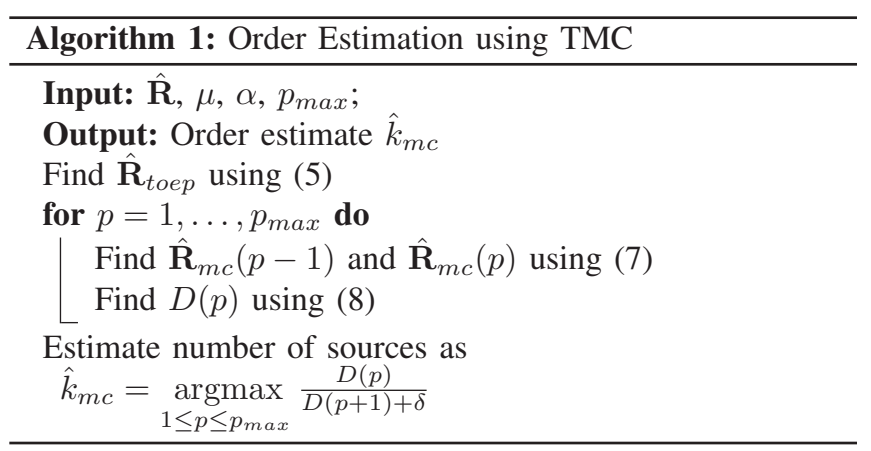

\section{Simulation Results}

We compare the performance of the proposed TMC with some representative methods for order estimation in the presence of non-iid noises and/or with low sample support, which are briefly reviewed in the following.

- Subspace Averaging (SA). By exploiting the shift invariance property of ULAs, the SA method estimates the low-dimensional signal subspace (and its dimension) as the average of a collection of subspaces extracted from consecutive sub-arrays [12], [13], [27]. The SA method, which was originally proposed for white noise and low sample support, was generalized to account for non-iid noises in [14] through a majority-vote approach.

- Minimum Description Length (MDL) for non-iid noises. The general expression of the MDL criterion is [4]

$\hat{k}_{M D L}=\underset{k=0, \ldots, M-1}{\operatorname{argmin}}\left\{-\log f\left(\mathbf{X} \mid \hat{\mathbf{R}}_{M L}\right)+\frac{1}{2} \nu_{k} \log N\right\}$,

where $\hat{\mathbf{R}}_{M L}$ denotes the Maximum Likelihood (ML) estimate for a covariance matrix with the required structure (low-rank plus diagonal) for a fixed order $k$, and $\nu_{k}=M+k(2 M-k)$ is the number of free-adjusted real parameters [28]. Although a closed-form ML estimate under non-iid noises is not possible, it can be obtained by using iterative algorithms [17], [18], [29].

- Linear Shrinkage- MDL (LSMDL). The standard MDL method proposed by Wax and Kailath [5] under the assumption of white noise is

$$
\begin{array}{r}
\hat{k}_{M D L}=\underset{0 \leq k \leq M-1}{\operatorname{argmin}}( \\
0-k) N \log \left(\frac{a(k)}{g(k)}\right) \\
+\frac{1}{2} k(2 M-k) \log N,
\end{array}
$$

where $a(k)$ and $g(k)$ are the geometric and the arithmetic mean, respectively, of the $M-k$ smallest eigenvalues of $\hat{\mathbf{R}}$. The linear shrinkage MDL (LS-MDL) [8], replaces the noise eigenvalues in the MDL criterion by a linear shrinkage.

For all simulations we assume that $K$ uncorrelated narrowband signals with a separation of $\Delta_{\theta}$ are impinging on an uniform linear array with $M$ half-wavelength separated 
antennas. For TMC, we use $\mu=\frac{M}{2}$ and $\alpha=\frac{M}{10}$. The signalto-noise-ratio is $\mathrm{SNR}=10 \log \frac{\operatorname{tr}\left(\mathbf{R}_{s}^{2}\right)}{\operatorname{tr}(\mathbf{\Sigma})}$.

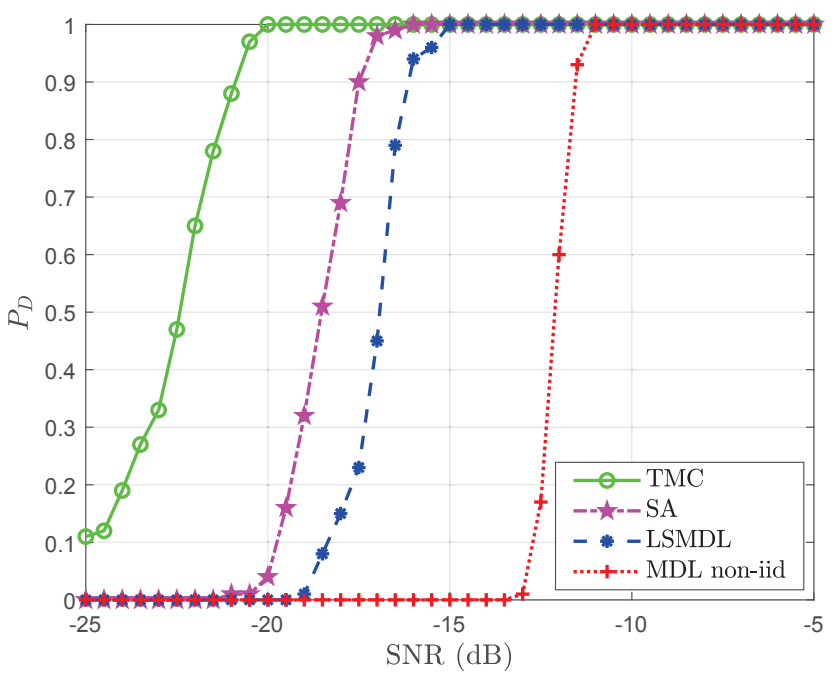

Fig. 2: $P_{D}$ vs. SNR for $M=100, N=150, K=3, \epsilon=0.3$, $\Delta_{\theta}=10^{\circ}$ and $\boldsymbol{\Psi}=\sigma_{s}^{2} \operatorname{diag}(1,0.8,0.6)$

Fig. 2 depicts the probability of correct detection $\left(P_{D}\right)$ with respect to SNR for an array of $M=100$ antennas, $K=3$ sources with $\Delta_{\theta}=10^{\circ}, N=150$ snapshots and $\epsilon=0.3$. Sources have unequal powers so that $\boldsymbol{\Psi}=\sigma_{s}^{2} \operatorname{diag}(1,0.8,0.6)$, where $\sigma_{s}^{2}$ is the common signal variance. We observe that TMC provides better performance than competing methods. Thanks to the Toeplitz rectification and the denoising of $\hat{\mathbf{R}}$, the TMC method reliably detects the rank of the signal covariance matrix at lower SNRs than the rest of methods and hence yields a more robust source enumeration method.

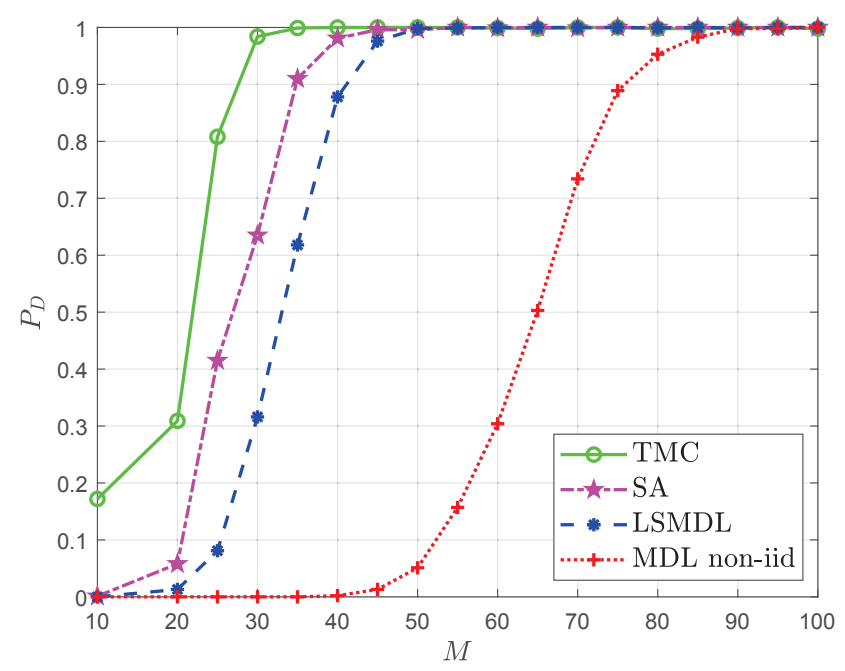

Fig. 3: $P_{D}$ vs. $M$ for $\frac{M}{N}=1, \Delta_{\theta}=\frac{2 \pi}{M}, K=3, \epsilon=0.3$ and $\mathrm{SNR}=-10 \mathrm{~dB}$ and sources have equal power
Fig. 3 shows $P_{D}$ vs. the number of sensors when $K=3$, $\epsilon=0.3, \mathrm{SNR}$ is fixed to $-10 \mathrm{~dB}$ and all sources have equal power. The ratio between the number of sensors and the number of snapshots is $\frac{M}{N}=1$ and the separation between sources is $\Delta_{\theta}=\frac{2 \pi}{M}$. This example shows that TMC provides accurate results in the small sample regime for arrays of varying number of sensors.

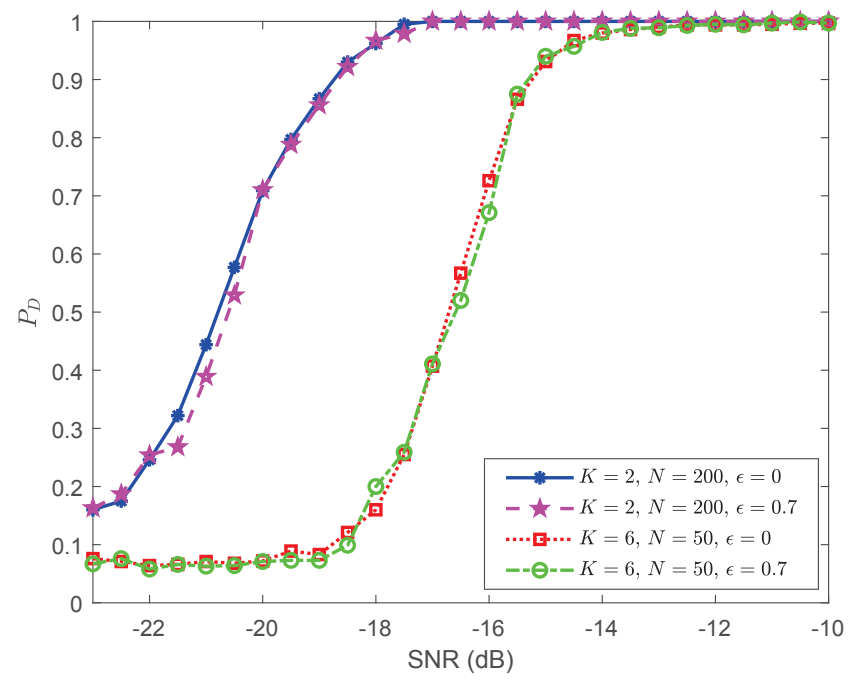

Fig. 4: $P_{D}$ vs. SNR for TMC when $M=50, \Delta_{\theta}=10^{\circ}$ and sources have equal power for different values of $K, N$ and $\epsilon$.

In the last experiment the robustness of the TMC method against the non-whiteness parameter, $\epsilon$, is examined in different scenarios. Since the diagonal terms of $\hat{\mathbf{R}}$ are eliminated as a pre-processing step, it is expected that the TMC results will not be affected by changes in $\epsilon$. This behavior can be observed for two different scenarios: i) $K=2$ and $N=200$ and ii) $K=6$ and $N=50$, in Fig. 4 , which shows $P_{D}$ with respect to SNR for $M=50, \Delta_{\theta}=10^{\circ}$ and equal power sources. It can be noticed that for both the scenarios, results are almost unaffected by $\epsilon$. Let us recall that $\epsilon=0$ represents the white noise case; therefore, TMC is robust under both uncorrelated non-iid and iid noises.

\section{Conclusions}

We addressed the problem of source enumeration when the noise covariance matrix is diagonal with unknown entries and with relatively few snapshots. The diagonal terms of the sample covariance matrix, which are most affected by this noise model, are eliminated and the off-diagonal terms are further denoised by Toeplitz rectification. The low-rank and Toeplitz signal covariance matrix is then reconstructed with matrix completion techniques. We have shown that the Frobenius norm of the signal covariance matrix reconstructed by the proposed denoising+MC technique provides an accurate order determination criterion. The method performs robustly for iid and non-iid noises, as well as for small and large arrays, in the small-sample regime. 


\section{REFERENCES}

[1] H. V. Trees, Detection, Estimation and Modulation Theory Part IV: Optimum Array Processing. New York, NJ: Wiley, 2002.

2] L. L. Scharf, Statistical Signal Processing: Detection, Estimation and Time Series Analysis. Reading, MA: Addison-Wesley, 1991.

[3] H. Akaike, "A new look at the statistical model identification," IEEE Trans. Autom. Control, vol. 19, no. 6, pp. 716-723, 1974.

[4] J. Rissanen, "Modeling by shortest data description," IEEE Trans. Acoust., Speech, Signal Process., vol. 14, pp. 465-471, 1978.

[5] M. Wax and T. Kailath, "Detection of signals by information theoretic criteria," IEEE Trans. Acoust., Speech, Signal Process., vol. 33, no. 2, pp. 387-392, 1985.

[6] Z. Lu and A. M. Zoubir, "Generalized Bayesian information criterion for source enumeration in array processing," IEEE Trans. Signal Process., vol. 61, no. 6, pp. 1470-1480, 2013.

[7] R. R. Nadakuditi and A. Edelman, "Sample eigenvalue based detection of high-dimensional signals in white noise with relatively few samples," IEEE Trans. Signal Process., vol. 56, no. 7, pp. 2625-2638, 2008

[8] L. Huang and H. C. So, "Source enumeration via MDL criterion based on linear shrinkage estimation of noise subspace covariance matrix," IEEE Trans. Signal Process., vol. 61, no. 19, pp. 4806-4821, 2013.

[9] L. Huang, Y. Xiao, H. C. So, and J.-K. Zhang, "Bayesian information criterion for source enumeration in large-scale adaptive antenna array," IEEE Trans. Veh. Technol., vol. 65, no. 5, pp. 3018-3032, 2016.

[10] A. Quinlan, J. Barbot, P. Larzabal, and M. Haartdt, "Model order selection for short data: An exponential fitting test (eft)," EURASIP Journal on Advances in Signal Processing, 2007.

[11] R. F. Brcich, A. M. Zoubir, and P. Pelin, "Detection of sources using bootstrap techniques," IEEE Trans. Signal Processing, vol. 50, pp. 206215, 2002.

[12] I. Santamaria, D. Ramirez, and L. L. Scharf, "Subspace averaging for source enumeration in large arrays," in Proc. IEEE Work. Stat. Signal Process. (SSP), Freiburg, Germany, Jun. 2018.

[13] V. Garg, I. Santamaria, D. Ramirez, and L. L. Scharf, "Subspace averaging and order determination for source enumeration," IEEE Trans. Signal Process., vol. 67, pp. 3028-3041, 2019.

[14] V. Garg and I. Santamaria, "Source enumeration in non-white noise and small sample size via subspace averaging," in 2019 27th European Signal Processing Conference (EUSIPCO), A Coruña, Spain, Sep. 2019.

[15] K. V. Mardia, J. T. Kent, and J. M. Bibby, Multivariate Analysis. New York: Academic, 1979.

[16] D. N. Lawley and A. E. Maxwell, Factor Analysis as a Statistical Method. American Elsevier Pub. Co., 1971.

[17] M. Pesavento and A. B. Gershman, "Maximum-likelihood direction-ofarrival estimation in the presence of unknown nonuniform noise," IEEE Trans. Signal Processing, vol. 49, no. 7, pp. 1310-1324, Jul. 2001

[18] D. Ramirez, G. Vázquez-Vilar, R. Lopez-Valcarce, J. Via, and I. Santamaria, "Detection of rank-p signals in cognitive radio networks with uncalibrated multiple antennas," IEEE Trans. Signal Process., vol. 59 no. 8, pp. 3764-3774, 2011.

[19] P. Vallet and P. Loubaton, "On the performance of MUSIC with Toeplitz rectification in the context of large arrays," IEEE Trans. Signal Processing, vol. 65, pp. 5848-5859, 2017.

[20] P. Forster, "Generalized rectification of cross spectral matrices for arrays of arbitrary geometry," IEEE Trans. Signal Processing, vol. 49, pp. 972978, 2001.

[21] P. Pal and P. P. Vaidyanathan, "A grid-less approach to underdetermined direction of arrival estimation via low rank matrix denoising," IEEE Signal Processing Letters, vol. 21, no. 6, pp. 737-741, 2014.

[22] W. Tan and X. Feng, "Covariance matrix reconstruction for direction finding with nested arrays using iterative reweighted nuclear norm minimization," Int. Journal of Antennas and Propagation, 2019

[23] B. Liao, C. Guo, L. Huang, and J. Wen, "Matrix completion based direction-of-arrival estimation in nonuniform noise," in 2016 IEEE International Conference on Digital Signal Processing (DSP)., Beijing, China, Oct. 2016

[24] E. J. Candes and Y. Plan, "Matrix completion with noise," Proc. of the IEEE, vol. 98, pp. 925-936, 2010.

[25] J. Vinogradova, R. Couillet, and W. Hachem, "Estimation of toeplitz covariance matrices in large dimensional regime with applications to source detection," IEEE Trans. Signal Process., vol. 63, pp. 4903-4913, 2019.
[26] N. Srebro, J. Rennie, and T. Jaakkola, "Maximum-margin matrix factorization," in Advances in Neural Information Processing Systems, Vancouver, Canada, Dec 2005, pp. 1329-1336.

[27] I. Santamaria, L. L. Scharf, M. Kirby, C. Peterson, and J. Francos, "An order fitting rule for optimal subspace averaging," in Proc. IEEE Work. Stat. Signal Process. (SSP), Palma de Mallorca, Spain, Jun. 2016.

[28] G. Vazquez-Vilar, D. Ramirez, R. Lopez-Valcarce, J. Via, and I. Santamaria, "Spatial rank estimation in cognitive radio networks with uncalibrated multiple antennas," in 4th International Conference on Cognitive Radio and Advanced Spectrum Management (cogART), Barcelona, Spain, Oct. 2011.

[29] K. G. Joreskog, "Some contributions to maximum likelihood factor analysis," psychometrika, vol. 32, pp. 443-482, 1967. 